\title{
Hand eczema severity and quality of life: a cross-sectional, multicentre study of hand eczema patients
}

Tove Agner ${ }^{1,2}$, Klaus E. Andersen ${ }^{3}$, Francisco M. Brandao $^{4}$, Derk P. Bruynzeel ${ }^{5}$, Magnus Bruze $^{6}$, Peter Frosch $^{7}$, Margarida Goncalo ${ }^{8}$, An Goossens ${ }^{9}$, Cristophe J. Le Coz $^{10}$, Thomas Rustemeyer ${ }^{5}$, Ian R. White ${ }^{11}$ and Thomas DIEPGen ${ }^{12}$, on behalf of the EECDRG*

${ }^{1}$ Department of Dermatology, Roskilde Hospital, University of Copenhagen, 4000 Roskilde, Denmark, ${ }^{2}$ Department of Dermatology, Gentofte Hospital, University of Copenhagen, 2900 Hellerup, Denmark, ${ }^{3}$ Department of Dermatology,

University of Southern Denmark, 5000 Odense, Denmark, ${ }^{4}$ Department of Dermatology, Hospital Garcia de Orta, 2805-267 Almada, Portugal, ${ }^{5}$ Department of Dermatology, Free University Hospital, NL-1081 HV Amsterdam, The Netherlands, ${ }^{6}$ Department of Occupational and Environmental Dermatology, Malmö University Hospital, Lund University, S-20502 Malmö, Sweden, ${ }^{7}$ Department of Dermatology, Klinikum Dortmund, University of Witten/ Herdecke, 44137 Dortmund, Germany, ${ }^{8}$ Department of Dermatology, University Hospital, P-3000-175 Coimbra, Portugal, ${ }^{9}$ Department of Dermatology, University Hospital, K. U. Leuven, 3000 Leuven, Belgium, ${ }^{10}$ Department of Dermatology, University Hospital, 67000 Strasbourg, France, ${ }^{11}$ Department of Cutaneous Allergy, St Thomas' Hospital, SE1 7EH London, UK, and ${ }^{12}$ Department of Social Medicine, University Hospital Heidelberg, 69115 Heidelberg, Germany

Background and Objectives: Hand eczema is a chronic disease with negative impact on quality of life (QoL). In this study, QoL in hand eczema patients is assessed and related to age, sex, severity, and diagnostic subgroups.

Methods: A total of 416 patients with hand eczema from 10 European patch test clinics participated in the study. Data on QoL were obtained from a self-administered questionnaire using the Dermatology Life Quality Index (DLQI). Severity was assessed by a scoring system (Hand Eczema Severity Index, HECSI) as well as frequency of eruptions and sick leave due to hand eczema.

Results: No significant difference was found between males and females with respect to QoL [DLQI median values and 25/75 percentiles for males and females being 7.0 (3-14) and 8.0 (3-13), respectively], although males were more severely affected than females $(P<0.025)$. A significant positive correlation was found for hand eczema severity and age $(P<0.001)$, while no significant correlation was found for QoL and age. QoL was found increasingly reduced when sick leave was getting higher $(P<0.001)$. A statistically significant correlation between QoL (as measured by DLQI) and hand eczema severity as measured by HECSI was found $(P<0.001)$. No significant difference in QoL was found between diagnostic subgroups.

Conclusions: QoL was found markedly negatively affected in hand eczema patients and was significantly correlated to disease severity. No significant difference in QoL was found between males and females, in spite of significantly more severe eczema in males, indicating that QoL in female patients is more easily affected.

Key words: hand eczema; HECSI; quality of life. (C) Blackwell Munksgaard, 2008.

Accepted for publication 28 February 2008

\footnotetext{
*European Environmental and Contact Dermatitis Research Group (EECDRG) (http://orgs.dermis.net/ content/e05eecdrg/index_ger.html).
}

Hand eczema is one of the most frequently seen diseases in dermatological practice. For one third of patients with hand eczema, the disease starts before 20 years of age (1), and it is often occupationally related (2). Due to its chronic course with 
mean disease duration of more than 10 years, hand eczema is an expensive disease for society and a burden for the individual $(3,4)$. Parameters such as severity, sick leave, and job loss may be used for assessment of the burden of the disease (5), but for the patient, the quality of life (QoL) is essential. Theoretically, QoL may be assessed by generic (global), dermatology-specific or diseasespecific questionnaires (6). However, for hand eczema, no disease-specific questionnaire has yet been developed, but both generic and dermatology-specific questionnaires have proved valuable $(7,8)$. Recent studies provide data showing equal impairment of QoL in patients with severe hand eczema and patients with atopic dermatitis and psoriasis (8). This study assesses QoL in hand eczema patients referred to dermatological clinics in different centres in Europe, relates QoL to disease severity and diagnostic subgroups of hand eczema, and identifies which factors influence QoL.

\section{Materials and Methods}

The study is a cross-sectional, multicentre study and was performed within the European Environmental and Contact Dermatitis Research Group (EECDRG) (http://orgs.dermis.net/content/ e05eecdrg/index_ger.html) in the period September 2005-August 2006. Patients with hand eczema referred to one of the 10 participating patch test clinics in Europe were invited to participate. Patients were included consecutively. Because participation in the study for the patients only meant to complete the QoL questionnaire, virtually all invited to participate in the study actually participated. Medical history including information about atopy, psoriasis, domestic and occupational exposures, frequency of eruptions, and sick leave was registered. All participants were patch tested with the European baseline series with 2 days occlusion and at least two readings, and underwent additional testing when needed. Finn Chambers $^{\mathbb{R}}$ (Epitest, Tuusula, Finland) were used in seven centres; in one centre, True test panels 1 and 2 were used additionally to Finn Chambers, and three centres used Van der Bend chamber (Brielle, the Netherlands) or IQ chamber (Chemotechnique, Vellinge, Sweden).

Data on QoL were obtained from a selfadministered questionnaire using the Dermatology Life Quality Index (DLQI) (9). This is a dermatology-specific questionnaire, which previously has proved useful for assessment of QoL in hand eczema patients $(8,10)$. The DLQI is a 10 -item questionnaire, which covers six aspects of daily life experienced during the past week: (i) symptoms and feelings, (ii) daily activities, (iii) leisure items, (iv) work and school, (v) personal relationship items, and (vi) treatment. The DLQI score is calculated by summing the score of each question, with a maximum score of 30 and a minimum score of 0 . The higher the score, the greater the impairment of life.

The clinical examination included evaluation of severity by use of a scoring system (Hand Eczema Severity Index, HECSI) (11). HECSI includes scoring of morphological symptoms including erythema, infiltration, vesicles, fissures, scaling, and oedema as well as a scoring of the affected area on the hands (fingertips, fingers, palms, back of hand, and wrist).

The diagnostic options, based on clinical practice and decided after discussion in the EECDRG group, were allergic contact dermatitis (ACD), irritant contact dermatitis (ICD), atopic hand eczema (AHE), vesicular hand eczema, and hyperkeratotic hand eczema (manuscript in preparation). The diagnoses ACD and ICD were used only when relevant allergic or irritant exposures were assessed. More than one diagnosis could be provided for each patient.

\section{Statistics}

For comparison between groups, the chi-squared test was used, and when more than two groups were included, the Kruskal-Wallis test was used. The Spearman correlation was used for correlation studies.

\section{Results}

A total of 416 patients from the 10 clinics were included, each clinic contributing 27-64 patients (4 clinics contributed 50 patients, 1 clinic 64 patients, and 5 clinics contributed less than 50 patients). Median age was 39 years (range 18-78 years), and participants were 252 females and 164 males. $23.5 \%$ had a personal history of atopic dermatitis (AD), 3.8\% reported psoriasis, and $63.0 \%$ had contact sensitization to one or more

Table 1. Median values (25/75 percentiles) for DLQI and HECSI given for males and females $(n=416)^{\mathrm{a}}$

\begin{tabular}{lrrrr}
\hline \multicolumn{2}{c}{ Total } & \multicolumn{1}{c}{ Males } & Females & $P$ value \\
\hline DLQI & $8.0(4.0-13.0)$ & $7.0(3.0-14.0)$ & $8.0(4.0-13.0)$ & 0.406 \\
HECSI & $17.0(8.0-35.0)$ & $20.5(10.0-51.0)$ & $14.5(6.0-30.0)$ & $<0.025$ \\
\hline
\end{tabular}

DLQI, Dermatology Life Quality Index; HECSI, Hand Eczema Severity Index.

${ }^{a}$ Higher score indicates more negative impairment on quality of life. Statistically significant higher HECSI score was found in males compared with females, while no significant difference was found for DLQI values. 
of the tested allergens. About $51.7 \%$ of all cases were reported as having occupational hand eczema.

DLQI and HECSI scores for assessment of QoL and hand eczema severity, respectively, are given in Table 1. Statistically significantly higher HECSI scores were found in males compared with females, while no significant difference was found for DLQI values (Table 1). The distribution of DLQI and HECSI scores in different age groups is given in Fig. 1. A significant positive correlation was found for HECSI and age $(P<0.001)$, while no significant correlation was found for DLQI and age $(P=0.11)$. Data for DLQI in relation to sick leave and frequency of eruptions are given
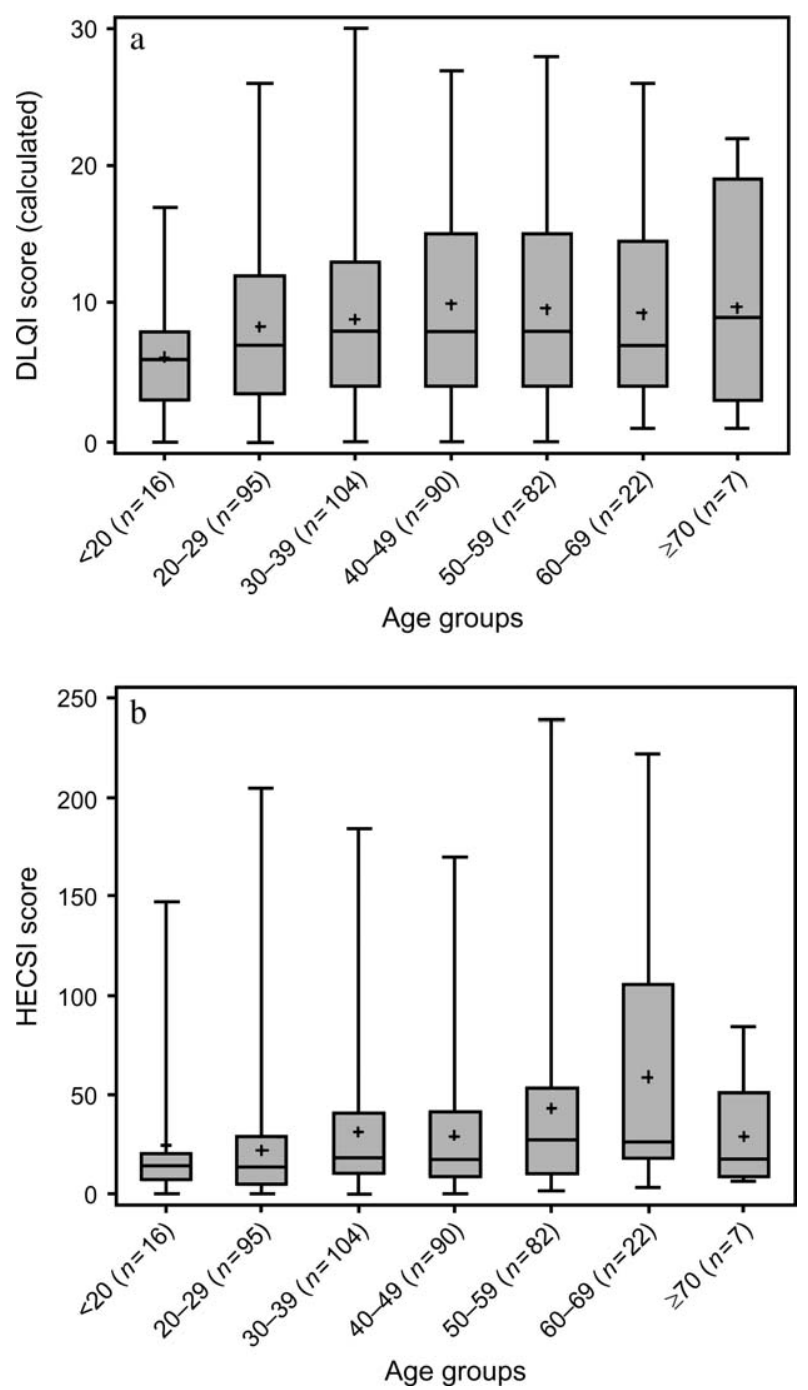

Fig. 1. DLQI (a) and HECSI (b) for the different age groups. Median values and 25/75 percentiles are given (boxes) as well as mean values $(+)$, maximum and minimum observations. No significant correlation was found for DLQI and age $(P=0.11)$, while a significant positive correlation was found for HECSI and age $(P<0.001)$. DLQI, Dermatology Life Quality Index; HECSI, Hand Eczema Severity Index.
Table 2. Median values and 25/75 percentiles for DLQI in relation to sick leave $(n=416)^{\mathrm{a}}$

\begin{tabular}{lr}
\hline Sick leave & \multicolumn{1}{c}{ DLQI } \\
\hline No sick leave $(n=299)$ & $6.0(3.0-12.0)$ \\
$<1$ week $(n=13)$ & $5.0(2.0-14.0)$ \\
$1-5$ weeks $(n=53)$ & $10.0(5.0-17.0)$ \\
$>5$ weeks $(n=51)$ & $13.5(8.0-18.0)$ \\
\hline
\end{tabular}

DLQI, Dermatology Life Quality Index.

${ }^{a}$ There was a statistically significant difference in DLQI between the groups with respect to sick leave $(P<0.001)$.

Table 3. Median values and 25/75 percentiles for DLQI in relation to number of eruptions $(n=414)^{\mathrm{a}}$

\begin{tabular}{ll}
\hline Eruptions* & DLQI \\
\hline Less than four eruptions/year $(n=42)$ & $6.0(1.0-11.0)$ \\
More than eruptions/year $(n=102)$ & $8.0(3.0-12.0)$ \\
Persistent dermatitis $(n=270)$ & $8.0(4.0-14.0)$ \\
\hline
\end{tabular}

DLQI, Dermatology Life Quality Index.

${ }^{a}$ There was a statistical trend for differences between the groups with respect to eruptions $(P=0.05)$.

in Tables 2 and 3. Increasing DLQI values, indicating lower QoL, were found with increasing sick leave $(P<0.001)$, and a trend was found for lower QoL with increasing number/duration of eruptions $(P=0.051)$.

A statistically significant positive correlation between DLQI and HECSI was found $(P<0.001)$, as shown in Fig. 2. For 316 patients, a precise diagnosis according to the given subgroups was made (manuscript in preparation), and DLQI values for these are given in Fig. 3. No statistically significant differences were found between the groups $(P=0.11)$, but there was a trend that QoL was most impaired in patients with ACD

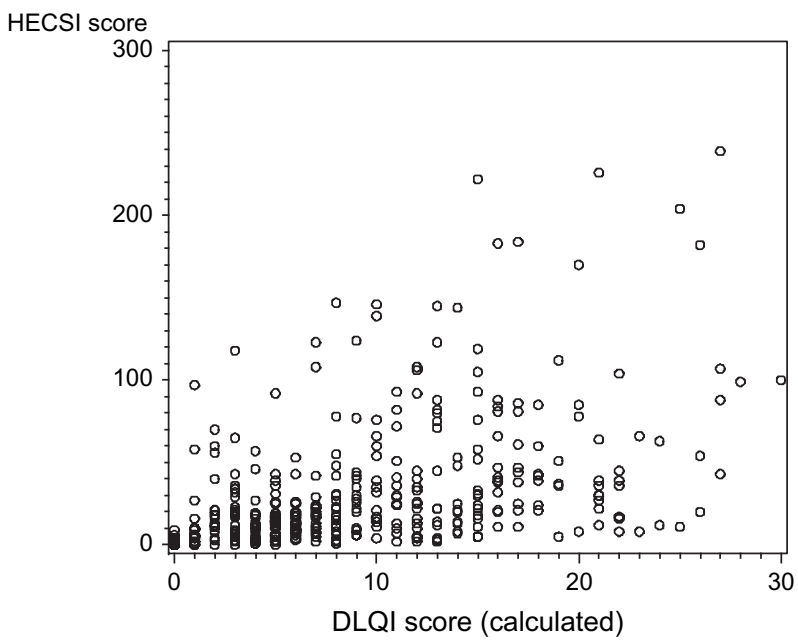

Fig. 2. A statistically significant positive correlation between Dermatology Life Quality Index and Hand Eczema Severity Index was found $(n=416, P<0.001)$. 


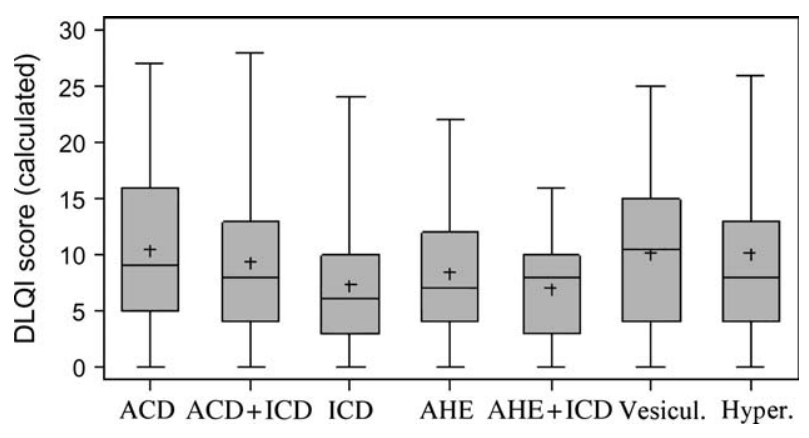

Fig. 3. DLQI score for diagnostic subgroups $(n=316)$. No significant difference was found between the groups $(P=$ 0.111 ). Median values and 25/75 percentiles are given (boxes) as well as mean values $(+)$, maximum and minimum observations. $A C D$, allergic contact dermatitis; $A H E$, atopic hand eczema; Hyper., hyperkeratotic hand eczema; ICD, irritant contact dermatitis; Vesicul., vesicular hand eczema.

and vesicular eczema and less affected in patients with ICD.

\section{Discussion}

In the present material of patients with hand eczema, referred to specialized European clinics, a median DLQI score of 8.0 was found, underlining that hand eczema is a diagnosis with significantly negative impact on QoL. The positive correlation between DLQI and HECSI shows a close relationship between QoL and disease severity and supports measurement of DLQI as a clinically relevant parameter. For the first time, QoL in relation to diagnostic subgroups of hand eczema is reported.

The study population was a typical cohort of hand eczema patients with respect to age and sex distribution. Contact sensitization was found in $63 \%$ of the population, which is higher than that reported in studies of hand eczema patients recruited from the background population (12), but in agreement with findings in patents with occupational hand eczema (13), which was diagnosed in half of the patients. The high sick leave frequency and the fact that more than $60 \%$ reported persistent dermatitis indicate that this population was severely affected. This was to be expected because the patients were referred to specialized dermatological clinics.

QoL was assessed by the DLQI. As opposed to generic questionnaires (e.g. the Short Form-36, SF-36), DLQI is a dermatology-specific questionnaire, which has proved useful for assessment of QoL among hand eczema patients. Comparing the efficacy of the DLQI and the generic SF-36 questionnaire for assessment of QoL in hand eczema favoured the SF-36, and a recent study found this to be a more accurate instrument in measuring mental health DLQI (7). However, the DLQI is a simpler and shorter questionnaire expected to give better compliance and was chosen for that reason. Ideally, a QoL questionnaire for hand eczema patients should include questions on work-related impairment of both physical functioning and interaction with colleagues (14), but such instruments are not at the moment available for this disease.

The DLQI median score of 8.0 was in agreement with previous findings in patients with severe occupational hand eczema, where DLQI was reported to be 7.8. (8), and comparable to DLQI values found in moderate psoriasis and atopic dermatitis.

Although females had less severe hand eczema than males, QoL was equally affected. In a previous study (7) using the SF-36 questionnaire, health-related QoL was reported more impaired for females with hand eczema than for males in the mental health dimension, whereas no sexrelated differences were detected with the DLQI, in agreement with our present findings. Another study in patients with ACD, using the Skindex-16 questionnaire for assessment of QoL, found no statistically significant sex-related differences in QoL scores, although also in this study females reported a higher degree of emotional distress $(15,16)$. The studies all indicate that QoL in female patients is more easily affected than in male patients. This may be related to the tradition that females take care of most domestic work and that this part of their life is being affected, or it may be related to the negative cosmetic influence of the disease, which also traditionally may be expected to cause more distress in females, and was recently reported to do so in female AD patients (17).

A positive correlation between HECSI and age illustrates that the hand eczema may become more severe in older age groups, while the QoL was not significantly affected with increasing age. In a recent study, more younger subjects with ACD reported a significantly injured QoL compared with older age groups (16), while no association between QoL and age was found in a population of patients with occupational hand eczema (8). Increased severity of the eczema but no significant worsening of QoL indicates that hand eczema may become more tolerated with increasing age.

The correlation between DLQI and HECSI, assessing the QoL and hand eczema severity, respectively, was expected and supports the relevance of both scoring systems. A correlation between severity and QoL in patients with occupational hand eczema has previously been reported $(8,10)$, but the positive correlation between HECSI and DLQI is reported in this study for the first 
time. Frequent eczema eruptions and long duration of sick leave contributed negatively to QoL, as would be expected. No significant differences in DLQI score between diagnostic subgroups of hand eczema was found, and this is in agreement with a previous study on occupational hand eczema where no difference in DLQI was reported between hand eczema patients diagnosed as allergic or irritant dermatitis or contact urticaria (8).

\section{Conclusions}

Our data confirm that patients with hand eczema, referred to specialized clinics for examination and treatment, have a significantly negatively affected QoL. Males and females had equally impaired QoL, although males had significantly more severe eczema. A significantly positive correlation between DLQI and disease severity was found, supporting the use of DLQI as a relevant clinical parameter.

\section{References}

1. Meding B, Jarvholm B. Incidence of hand eczema - a population-based retrospective study. $J$ Invest Dermatol 2004: 122: 873-877.

2. Funke U, Fartasch M, Diepgen T L. Incidence of workrelated hand eczema during apprenticeship: first results of a prospective cohort study in the car industry. Contact Dermatitis 2001: 44: 166-172.

3. Meding B. Epidemiology of hand eczema in an industrial city. Acta Derm Venereol 1990: 153 (Suppl.): 1-43.

4. Meding B, Swanbeck G. Consequences of having hand eczema. Contact Dermatitis 1990: 23: 6-14.

5. Cvetkovski R S, Zachariae R, Jensen H, Olsen J, Johansen J D, Agner T. Prognosis of occupational hand eczema: a followup study. Arch Dermatol 2006: 142: 305-311.

6. Skoet R, Zachariae R, Agner T. Contact dermatitis and quality of life: a structured review of the literature. $B r J$ Dermatol 2003: 149: 452-456.
7. Wallenhammar L M, Nyfjall M, Lindberg M, Meding B. Health-related quality of life and hand eczema - a comparison of two instruments, including factor analysis. $J$ Invest Dermatol 2004: 122: 1381-1389.

8. Cvetkovski R S, Zachariae R, Jensen H, Olsen J, Johansen J D, Agner T. Quality of life and depression in a population of occupational hand czema patients. Contact Dermatitis 2006: 54: 106-111.

9. Finlay A Y, Khan G K. Dermatology Life Quality Index (DLQI) - a simple practical measure for routine clinical use. Clin Exp Dermatol 1994: 19: 210-216.

10. Thomson K F, Wilkinson S M, Sommer S, Pollock B. Eczema: quality of life by body site and the effect of patch testing. Br J Dermatol 2002: 146: 627-630.

11. Held E, Skoet R, Johansen J D, Agner T. The hand eczema severity index (HECSI): a scoring system for clinical assessment of hand eczema. A study of inter- and intra-observer reliability. Br J Dermatol 2005: 152: 302-307.

12. Nielsen N H, Linneberg A, Menne T, Madsen F, Frølund L, Dirksen A, Jørgensen T. The association between contact allergy and hand eczema in 2 cross-sectional surveys 8 years apart. Contact Dermatitis 2002: 47: 71-77.

13. Skoet R, Olsen J, Mathiesen B, Iversen L, Johansen J D, Agner T. A survey of occupational hand eczema in Denmark. Contact Dermatitis 2004: 51: 159-166.

14. Coenraads P J, Bouma J, Diepgen T L. Quality of life of patients with occupationally-induced hand eczema. Hautarzt 2004: 55: 28-30.

15. Kadyk D L, McCarter K, Aachen F, Belsito D V. Quality of life in patients with allergic contact dermatitis. $\mathrm{J} \mathrm{Am} \mathrm{Acad}$ Dermatol 2003: 49: 1037-1048.

16. Kadyk D L, Hall S, Belsito V. Quality of life of patients with allergic contact dermatitis: an exploratory analysis by gender, ethnicity, age, and occupation. Dermatitis 2004: 15: $117-124$.

17. Holm E A, Esmann J, Jemec G. Does visible atopic dermatitis affect quality of life more in women than in men? Gend Med 2004: 1: 125-130.

Address:

Tove Agner MD, PhD

Department of Dermatology

Roskilde Hospital

4000 Roskilde

Denmark

e-mail: t.agner@dadlnet.dk 